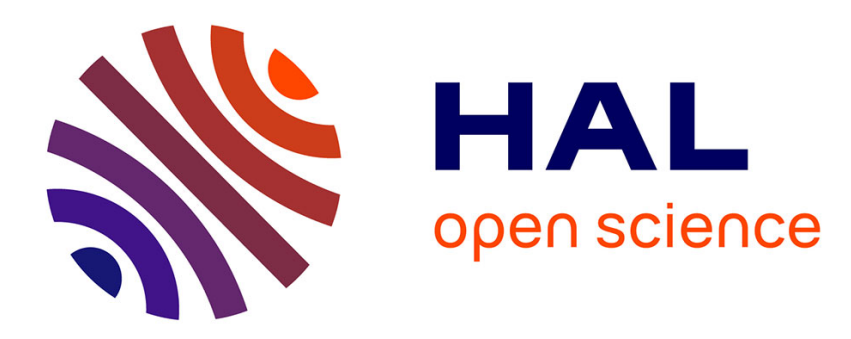

\title{
Influence de l'ablation des parois sur les caractéristiques d'un arc électrique laminé
}

\author{
B. Cheminat
}

\section{To cite this version:}

B. Cheminat. Influence de l'ablation des parois sur les caractéristiques d'un arc électrique laminé. Revue de Physique Appliquée, 1989, 24 (3), pp.277-284. 10.1051/rphysap:01989002403027700 . jpa00246049

\section{HAL Id: jpa-00246049 https://hal.science/jpa-00246049}

Submitted on 1 Jan 1989

HAL is a multi-disciplinary open access archive for the deposit and dissemination of scientific research documents, whether they are published or not. The documents may come from teaching and research institutions in France or abroad, or from public or private research centers.
L'archive ouverte pluridisciplinaire HAL, est destinée au dépôt et à la diffusion de documents scientifiques de niveau recherche, publiés ou non, émanant des établissements d'enseignement et de recherche français ou étrangers, des laboratoires publics ou privés. 


\title{
Influence de l'ablation des parois sur les caractéristiques d'un arc électrique laminé
}

\author{
B. Cheminat $\left({ }^{1}\right)$ \\ Télémécanique Electrique, 33 bis, Avenue du Maréchal-Joffre, B.P. 204, 92002 Nanterre, France \\ (1) Laboratoire de Physique des Matériaux, Université Blaise Pascal (UA 828), 63177 Aubière Cedex, France
}

(Reçu le 10 novembre 1987, révisé le 18 juillet 1988, accepté le 26 septembre 1988)

\begin{abstract}
Résumé. - L'étude expérimentale des interactions entre l'arc électrique et les parois du boîtier des appareils de coupure montre l'importance de la géométrie de la chambre de décharge et conduit à la sélection de matériaux.
\end{abstract}

\begin{abstract}
An experimental investigation was carried out on the interactions between an electric arc and the walls of the casings of breakers apparatus. Throughout the experiment, the discharge chamber design was shown to be very important, therefore making it necessary to select the materials carefully.
\end{abstract}

\section{Introduction.}

L'utilisation industrielle de nouvelle technique de coupure en basse tension est à l'origine de cette étude sur les arcs laminés à parois ablatives. La technique de limitation du courant [1] n'est plus basée sur l'allongement rapide de l'arc entre les contacts mais sur le laminage de cet arc entre deux pièces diélectriques. L'interaction arc-parois est forte. La présence de vapeurs issues de ces parois modifie profondément les caractéristiques du plasma. L'arc s'éteint rapidement.

Les avantages de cette nouvelle méthode de limitation du courant sont nombreux [1]. Les performances obtenues sont comparables à celles des fusibles.

L'étude expérimentale présentée concerne essentiellement les caractéristiques électriques de l'arc (courant-tension-champ électrique). Des mesures de pression ont également été faites. Les conditions expérimentales choisies permettent de mettre en évidence des différences de comportement importantes entre les matériaux.

L'usage industriel de cette technique a conduit à utiliser des produits commerciaux courants. De nombreux matériaux ont été essayés. Les polymères se sont, dès les premiers essais, révélés les plus prometteurs, aussi constituent-ils la majorité des échantillons.
La recherche bibliographique concernant des décharges d'arc avec ablation des parois montre que peu de travaux ont été faits sur le sujet. En raison d'importantes différences tant d'objectifs que de conditions expérimentales, aucun des résultats obtenus au cours de cette étude n'a pu être comparé à ceux d'autres auteurs. On peut néanmoins citer les modèles d'arc cylindrique proposés par Niemeyer [2], Ibrahim [3] et Kovitya [4] ainsi que l'étude de Tslaf $[5,6]$.

\section{Le dispositif expérimental.}

Le dispositif comprend la chambre à arc et le circuit d'alimentation ainsi que les ensembles de mesure du champ électrique, de la pression et de la vitesse d'expansion de l'arc.

2.1 LA CHAMBRE A ARC ET LE CIRCUIT D'ALIMENTATION. - L'arc se forme dans l'air dans une fente étroite entre deux électrodes en cuivre. La maquette de la chambre à arc est représentée sur la figure 1. La chambre est montée sur un support en bakélite fixé au banc d'essai. Les électrodes, distantes de $25 \mathrm{~mm}$, sont vissées sur le support.

La chambre à arc (Fig. 2) comporte deux joues latérales constituées du matériau à étudier. L'écartement est réglé à l'aide d'un système de cales. La largeur de la fente peut varier de $0,55 \mathrm{~mm}$ à $1 \mathrm{~mm}$. 


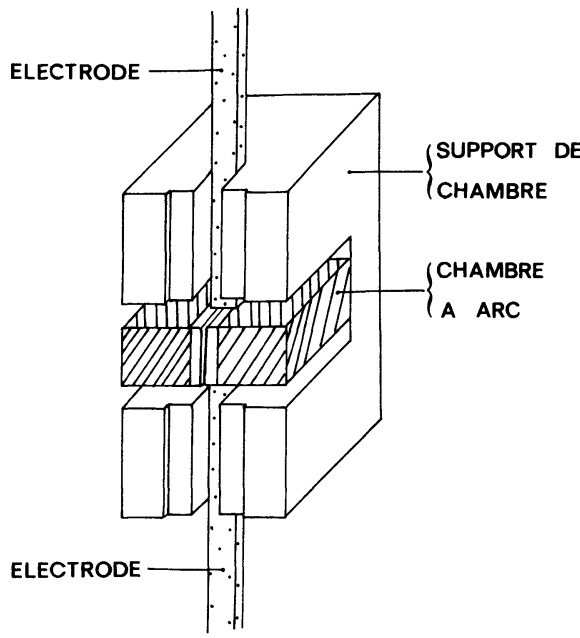

Fig. 1. - La chambre à arc.

[The arc-chamber.]

Une encoche en face avant permet de glisser la lamelle transparente assurant la fermeture de la chambre. Des essais faits avec des lamelles en verre et en plexiglas montrent que seul le plexiglas reste transparent en cours de décharge. C'est ce matériau qui a été retenu, tenant compte pour ce choix de la moindre fragilité des lamelles et des perspectives d'analyse spectrométrique de la décharge. Par ailleurs l'influence de la nature du matériau constituant la lamelle est négligeable. L'emplacement du capteur de pression et celui des sondes de mesure du champ électrique sont indiqués (Fig. 2).

Le circuit d'alimentation comprend un ensemble de condensateurs $(C=0,015 \mathrm{~F})$ décrit par Laurent [7]. La tension de charge varie de $460 \mathrm{~V}$ à $520 \mathrm{~V}$ selon les essais. L'inductance mise en série avec l'arc a une valeur de l'ordre de $38 \mu \mathrm{H}$. Une sonde à effet Hall du type LEM LA 1500 T5, en série avec l'arc, permet la mesure du courant d'arc. L'intensité de ce dernier dépend beaucoup des conditions expérimentales. Elle peut atteindre $4000 \mathrm{~A}$. La tension d'arc est mesurée entre les deux électrodes. L'amorçage se fait par l'intermédiaire d'un fil fusible placé entre les électrodes.

Pour un circuit extérieur donné, l'intensité du courant d'arc dépend de la nature des parois et de la largeur de la fente. La tension de charge est limitée à $520 \mathrm{~V}$. Le banc de condensateurs ne permet pas de faire des mesures à intensité constante pour différentes largeurs de fente et divers matériaux de paroi. Ceci rend délicate l'interprétation de certains résultats et interdit toute mesure pour des valeurs de la largeur de fente inférieure à $0,55 \mathrm{~mm}$. En effet, pour certains matériaux et de très faibles largeurs de fente, la décharge se limite pratiquement à l'explosion du fil fusible. Ceci est lié à la fois à la pression dans la chambre à arc et à la nature des vapeurs provenant des parois.

2.2 Mesure DU ChAMP ÉleCtRiQue. - On utilise deux sondes en tungstène de $0,8 \mathrm{~mm}$ de diamètre qui sont implantées dans la chambre comme l'indique la figure 2. Elles affleurent la paroi et sont distantes de $8 \mathrm{~mm}$.

En supposant la différence de potentiel sondeplasma égale pour les deux sondes et le champ électrique constant entre les deux sondes on peut déterminer ce dernier à partir de la valeur de la différence de potentiel entre sondes.

2.3 Mesure de la pression. - Le capteur de pression est un capteur piézoélectrique Kistler $601 \mathrm{~A}$ (gamme 1-250 bars) de $5,5 \mathrm{~mm}$ de diamètre. Il est placé dans la paroi de la chambre à arc (Fig. 2) et associé à un amplificateur.

2.4 Mesure de LA VITESSE D'EXPANSION DE l'ARC. - Deux fibres optiques distantes de $8 \mathrm{~mm}$ sont pour cette mesure implantées dans la paroi (Fig. 2a). L'extrémité de chaque fibre est reliée à un phototransistor et à un amplificateur. Le signal est ensuite transféré vers la chaîne d'acquisition de données. La vitesse d'expansion est calculée à partir du temps qui sépare l'apparition des deux signaux.

Le système d'acquisition de données a quatre voies d'entrées. Les signaux sont codés sur 8 bits et

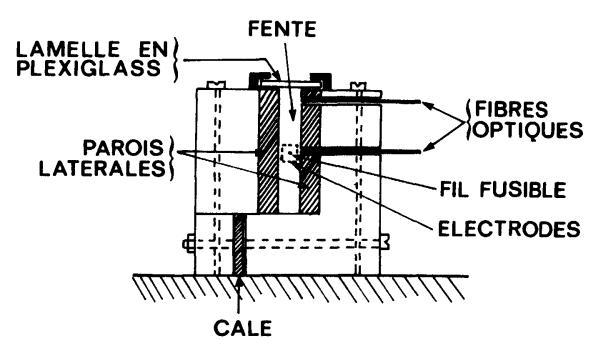

(a)

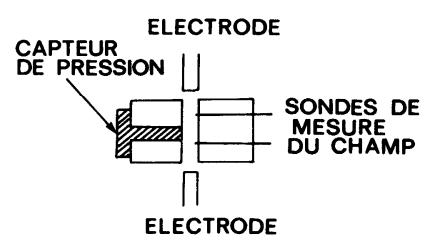

(b)

Fig. 2. - Vue en coupe de la chambre à arc. a) transversale; b) longitudinale.

[A cross-section of the arc-chamber. a) traverse section ; b) longitudinal section.] 
échantillonnés sur 4096 points. La fréquence d'échantillonnage est de $2 \mathrm{MHz}$. Ce système est piloté par un ordinateur qui assure également le transfert et la mémorisation des données ainsi que le traçage des courbes.

\section{Les matériaux.}

Les matériaux concernés par cette étude sont des polymères, des matériaux composites à base de polymères et une céramique d'origine commerciale. Ils sont présentés dans le tableau I. Leur composition est celle donnée par le fabricant, elle ne tient pas compte des additifs (antifeu, durcisseur, agent de démoulage). L'influence des colorants sera considérée lors de l'analyse des résultats.

\section{Les résultats.}

4.1 COURANT, TENSION, PUISSANCE ET RÉSISTANCE D'UN ARC LAMINÉ. - La figure 3 représente l'évolution en fonction du temps, du courant, de la tension, de la puissance et de la résistance d'un arc laminé entre des parois en PMMA distantes de $1 \mathrm{~mm}$. L'arc dure $3,5 \mathrm{~ms}$. La forme du courant est assez proche d'une demi-sinusoïde. On remarque au début de la décharge l'explosion du fil fusible. La courbe d'évolution de la tension présente un palier à environ $300 \mathrm{~V}$. La puissance varie comme le courant. La variation de résistance est continue.

Ces courbes sont à comparer à celles de la figure 4 représentant l'évolution des mêmes grandeurs lorsque l'arc n'est pas laminé. La chambre à arc ayant été enlevée, un fil fusible est placé entre les électrodes disposées comme précédemment à $25 \mathrm{~mm}$ l'une de l'autre. Pour une puissance équivalente, l'intensité du courant est élevée et la tension d'arc faible. Ceci montre l'intérêt du laminage de l'arc en vue de favoriser son extinction dans un appareillage de coupure.
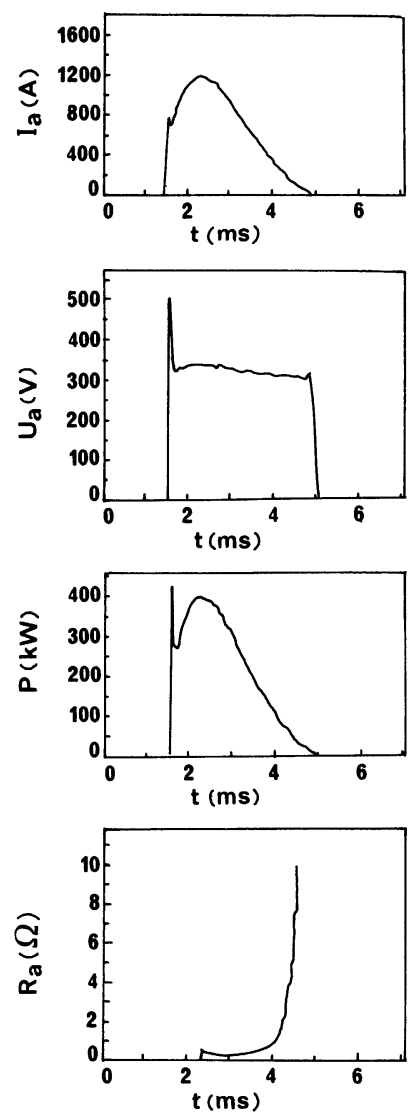

Fig. 3. - Courant, tension, puissance et résistance d'un arc laminé.

[Current, voltage, power and resistance of a laminated arc.]

Lors de tous les essais faits les courbes obtenues ont une allure similaire à celles de la figure 3. Aussi, seule, parmi ces 4 courbes, celle qui représente l'intensité du courant d'arc en fonction du temps a été systématiquement enregistrée.

Tableau I. - Les matériaux.

[The materials.]

\begin{tabular}{|c|c|c|}
\hline Catégorie & Abréviation & Composition \\
\hline $\begin{array}{l}\text { Céramique } \\
\text { Thermoplastique } \\
\text { Thermoplastique } \\
\text { Thermoplastique } \\
\text { Thermoplastique } \\
\text { Thermoplastique } \\
\text { Thermodurcissable } \\
\text { Composite } \\
\text { Composite } \\
\text { Composite }\end{array}$ & $\begin{array}{l}\text { ST } \\
\text { PTFE } \\
\text { PMMA } \\
\text { PA } \\
\text { PA 66 } \\
\text { PETP } \\
\text { PU } \\
\text { PAFV } 1 \\
\text { PAFV } 2 \\
\text { AR }\end{array}$ & $\begin{array}{c}\mathrm{SiO}_{2}(47 \%) \mathrm{Al}_{2} \mathrm{O}_{3}(40 \%) \mathrm{MgO}(10 \%) \\
\text { polytétrafluoroéthylène } \\
\text { polyméthacrylate de méthyle } \\
\text { polyacétal } \\
\text { polyamide } 6-6 \\
\text { polyéthylènetéréphtalate } \\
\text { polyuréthane } \\
\text { polyamide } 6-6(72 \%) \text { fibre de verre }(25 \%) \\
\text { polyamide } 6-6(72 \%) \text { fibre de verre }(25 \%) \text { colorant noir } \\
\text { PETP }(67 \%) \text { fibre de verre }(33 \%)\end{array}$ \\
\hline
\end{tabular}



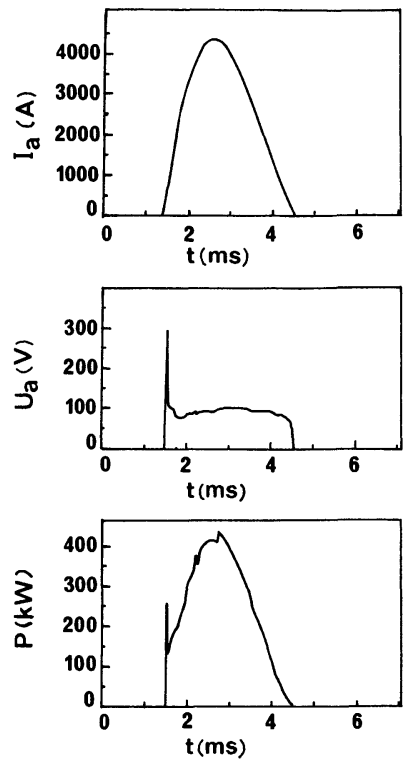

Fig. 4. - Courant, tension et puissance d'un arc non laminé.

[Current, voltage and power of a non-laminated arc.]

De nombreux essais ont montré que la chambre pouvait, sauf dans le cas du PTFE, être utilisée pour 4 décharges sans variations importantes des caractéristiques de l'arc. Les parois latérales doivent ensuite être changées.

L'expérimentation sur des matériaux différents avec un circuit extérieur identique (la tension de charge des condensateurs est de $520 \mathrm{~V}$ ) et la même largeur de fente $\left(l_{\mathrm{f}}=0,65 \mathrm{~mm}\right)$ conduit à des valeurs de l'intensité du courant qui peuvent être très différentes. A titre d'exemple, les courbes de la figure 5 correspondent à la variation de l'intensité pour les matériaux PA, PTFE, ST et AR. On peut noter les faibles valeurs de l'intensité obtenues pour PA et AR. L'analogie des courbes obtenues pour la céramique et le PTFE sera discutée par la suite.

Pour un même circuit, l'intensité du courant décroît lorsque la largeur de fente diminue. Les résultats obtenus pour le composite AR dont la composition est donnée dans le tableau I, ont été reportés sur la figure 6. L'observation de ces courbes montre la grande influence de la largeur de fente.

En prenant pour grandeur de référence l'intensité maximale du courant d'arc dans des conditions expérimentales fixées, on peut distinguer deux catégories de matériaux :

- la céramique et le PTFE. L'intensité maximale de courant est d'environ $1600 \mathrm{~A}$;

— les autres. L'intensité est alors de 600 à $900 \mathrm{~A}$.

La mesure du champ électrique permet de distinguer les matériaux au sein de chaque catégorie.

4.2 Le ChAMP ÉleCtriQue. - Les courbes de la figure 7 montrent l'évolution du champ électrique en fonction du temps dans deux cas différents :

- la fente est large $(1 \mathrm{~mm})$, le champ électrique relativement faible et l'intensité du courant élevée ;

- la fente est étroite $(0,55 \mathrm{~mm})$. En raison de la nature des vapeurs provenant des parois, le champ électrique est élevé et l'intensité du courant faible.

Dans les deux cas, on peut remarquer le pic correspondant à la fusion du fil fusible. La courbe présente un palier dont la valeur sera relevée pour différents matériaux et plusieurs valeurs de la largeur de la fente.

Les courbes de la figure 8 représentent la variation du champ électrique en fonction de la largeur de fente pour l'ensemble des matériaux considérés à
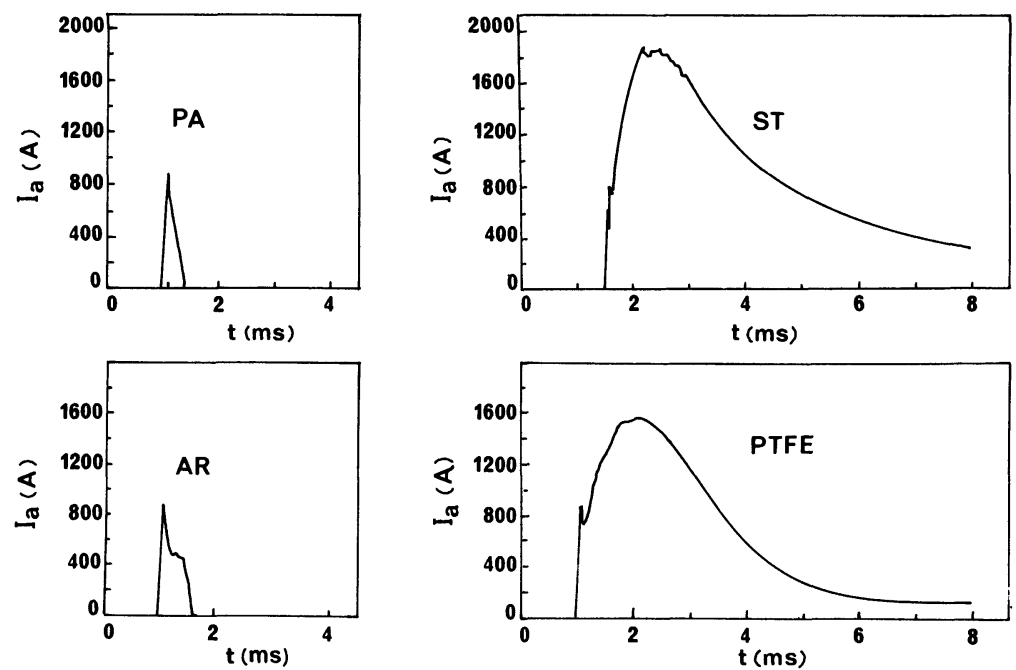

Fig. 5. - Evolution du courant dans un arc laminé entre des parois de nature diverse distantes de 0,65 mm.

[Current evolution in a laminated arc between walls of different kinds $0.65 \mathrm{~mm}$ apart.] 


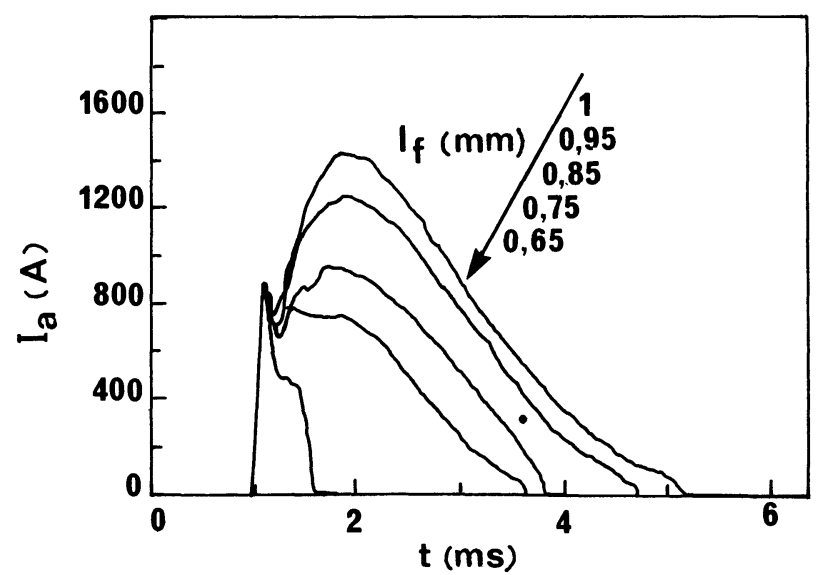

Fig. 6. - Variation en fonction du temps de l'intensité de courant d'un arc laminé entre des parois en AR pour différentes valeurs de la largeur de fente.

[Variations in terms of time of the current intensity of a laminated arc between AR walls for different values of the slit width.]
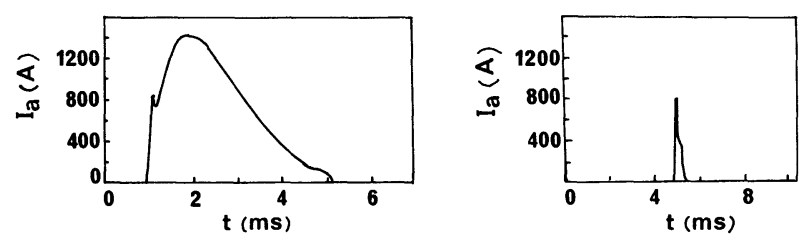

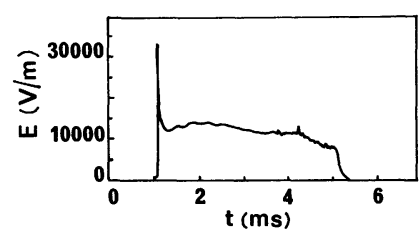

(a)

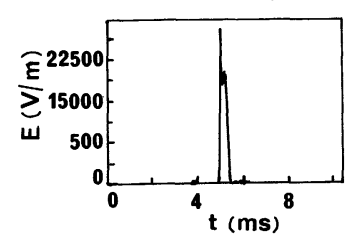

(b)
Fig. 7. - Evolution du champ électrique en fonction du temps. a) $l_{\mathrm{f}}=1 \mathrm{~mm}$; b) $l_{\mathrm{f}}=0,55 \mathrm{~mm}$.

[Evolution of the electric field in terms of time. a) s.w. $=1 \mathrm{~mm}$; b) s.w. $=0.55 \mathrm{~mm}$.]

l'exception de la céramique et du PTFE. Ces résultats apparaissent également dans le tableau II où ont été reportées aussi les valeurs de l'intensité de courant d'arc.

La céramique et le PTFE présentent des caractéristiques particulières. L'intensité du courant reste élevée même pour de faibles valeurs de la largeur de fente $(1600 \mathrm{~A}$ à $0,65 \mathrm{~mm})$. La valeur du champ électrique est faible $(11000 \mathrm{~V} / \mathrm{m}$ pour le PTFE; $15000 \mathrm{~V} / \mathrm{m}$ pour la céramique).

4.3 VITESSE D'EXPANSION DE L'ARC. - La vitesse d'expansion de l'arc a été mesurée pour PAFV2 avec le dispositif à sondes optiques décrit. La largeur de la fente est de $1 \mathrm{~mm}$. La moyenne de plusieurs essais

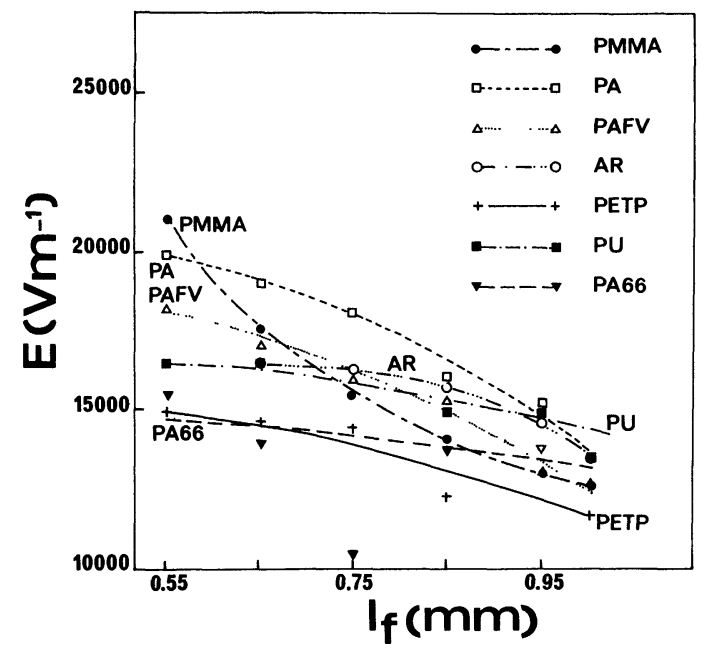

Fig. 8. - Variation du champ électrique en fonction de la largeur de fente pour différents matériaux.

[Variations of the electric field according to the slit width for different materials.]

conduit à une vitesse d'expansion de $600 \mathrm{~m} / \mathrm{s}$. Cette mesure permet de vérifier que le temps d'expansion $(\sim 13 \mu \mathrm{s})$ est faible devant la durée du phénomène (quelques millisecondes). Cette phase de la décharge peut être négligée.

4.4 LA PRESSION. - La pression est mesurée à l'aide d'un capteur piézoélectrique dont la surface sensible affleure, sous un adhésif noir, la paroi de la chambre à arc. La figure 10 représente l'évolution de la pression dans la chambre de décharge en fonction du temps. On peut remarquer au début de la décharge l'importante surpression correspondant à l'explosion $\mathrm{du}$ fil fusible. La pression moyenne correspondant au palier a été mesurée pour certains matériaux et différentes valeurs de la largeur de fente. Les résultats apparaissent dans le tableau III. $\mathrm{U}(\mathrm{V})$ représente la tension de charge des condensateurs. Elle est dans certains cas limitée à $450 \mathrm{~V}$ afin de réduire l'intensité du courant d'arc. La précision sur la mesure de pression est estimée à $5 \%$.

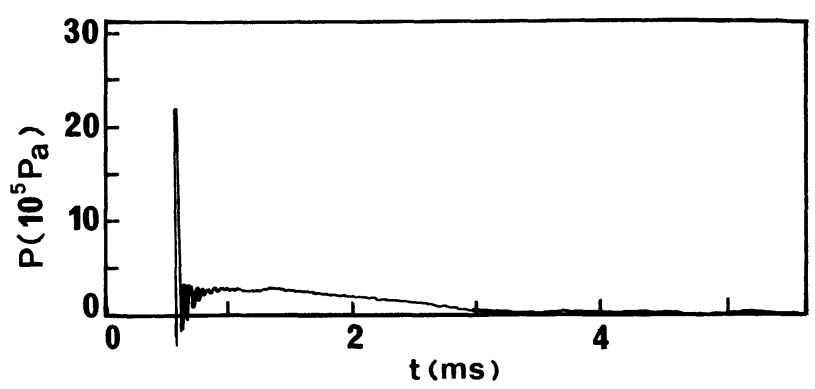

Fig. 10. - Evolution de la pression en fonction du temps.

[Pressure variation in terms of time.] 
Tableau II. - Champ électrique et courant d'arc pour différents matériaux et plusieurs largeurs de fente. [Electric field and arc-current for different materials and several slit widths.]

\begin{tabular}{|l|c|c||c|c||c|c|}
\hline \multirow{2}{*}{$l_{\mathrm{f}}(\mathrm{mm})$} & \multicolumn{2}{|c||}{ PA } & \multicolumn{2}{c||}{ PA 6-6 } & \multicolumn{2}{c|}{ PMMA } \\
\cline { 2 - 7 } & $E\left(\mathrm{Vm}^{-1}\right)$ & $I(\mathrm{~A})$ & $E\left(\mathrm{Vm}^{-1}\right)$ & $I(\mathrm{~A})$ & $E\left(\mathrm{Vm}^{-1}\right)$ & $I(\mathrm{~A})$ \\
\hline 0,55 & 19900 & 910 & 15500 & 950 & 21000 & 600 \\
0,65 & 19000 & 900 & 14000 & 1200 & 17500 & 700 \\
0,75 & 18100 & 860 & 10500 & 1000 & 15500 & 900 \\
0,85 & 16000 & 890 & 13800 & 1200 & 14000 & \\
0,95 & 15200 & 890 & 13800 & 1200 & 13000 & 1400 \\
1 & 13500 & 910 & & & 12500 & 1400 \\
\hline
\end{tabular}

\begin{tabular}{|l|c|c||c|c||c|c|}
\hline \multirow{2}{*}{$l_{\mathrm{f}}(\mathrm{mm})$} & \multicolumn{2}{|c||}{$\mathrm{PU}$} & \multicolumn{2}{c||}{ AR } & \multicolumn{2}{c|}{ PETP } \\
\cline { 2 - 6 } & $E\left(\mathrm{Vm}^{-1}\right)$ & $I(\mathrm{~A})$ & $E\left(\mathrm{Vm}^{-1}\right)$ & $I(\mathrm{~A})$ & $E\left(\mathrm{Vm}^{-1}\right)$ & $I(\mathrm{~A})$ \\
\hline 0,55 & 16600 & 800 & & & 15000 & 520 \\
0,65 & 16600 & 900 & 16500 & 450 & 14600 & 870 \\
0,75 & 14500 & 1270 & 16200 & 750 & 14500 & 1200 \\
0,85 & 15000 & 1250 & 15800 & 900 & 12300 & 1200 \\
0,95 & 15000 & 1200 & 14600 & 1200 & & \\
1 & & & 13500 & 1400 & 11700 & 1200 \\
\hline
\end{tabular}

\begin{tabular}{|l|c|c||c|c|}
\hline \multirow{2}{*}{$l_{\mathrm{f}}(\mathrm{mm})$} & \multicolumn{2}{|c||}{ PAFV 2 } & \multicolumn{2}{c|}{ PAFV 1 } \\
\cline { 2 - 5 } & $E\left(\mathrm{Vm}^{-1}\right)$ & $I(\mathrm{~A})$ & $E\left(\mathrm{Vm}^{-1}\right)$ & $I(\mathrm{~A})$ \\
\hline 0,55 & & & 18200 & 890 \\
0,6 & 16000 & 1100 & & \\
0,65 & 15000 & 1200 & 17200 & 890 \\
0,75 & 14000 & 1300 & 16000 & 890 \\
0,80 & 12000 & 1400 & 15300 & 1100 \\
0,85 & 10000 & 1500 & 13000 & 1280 \\
0,90 & 12500 & 1280 \\
0,95 & & & & \\
1 & & &
\end{tabular}

L'expérimentation est délicate en raison des risques d'amorçage sur le capteur. On peut noter que pour tous les matériaux à l'exception du polyacétal, la pression augmente lorsque la largeur de fente diminue. Ce phénomène peut être lié à l'ablation des parois et/ou à la variation de la viscosité dynamique du milieu formé dans la chambre de décharge (l'écoulement aux extrémités se ferait différemment).

4.5 INFLUENCE DES ADDITIFS. - Des essais ont été faits avec trois polyacétals d'origine différente. Ces matériaux, commercialisés comme polyacétal par des fabricants différents, peuvent différer légèrement en raison des additifs de fabrication : durcisseur, agent de démoulage, colorant. L'un de ces matériaux est blanc, les deux autres sont noirs. La courbe de la figure 11 représente l'évolution du champ électrique en fonction de la largeur de fente pour les trois matériaux. Les valeurs sont similaires. On peut cependant remarquer les valeurs légèrement supérieures obtenues avec un polyacétal blanc $(+)$ pour de faibles largeurs de fente.

En ce qui concerne les matériaux PAFV1 et PAFV2, dont la composition est donnée dans le tableau I, le phénomène est plus accentué. La valeur du champ électrique est notablement différente pour les deux matériaux comme le montrent les courbes 
Tableau III. - Variation de la pression en fonction de la largeur de fente pour différents matériaux.

[Pressure variation for different materials, depending on the slit width.]

\begin{tabular}{|c|c|r|r|c|}
\hline matériau & $l_{\mathrm{f}}(\mathrm{mm})$ & $U(\mathrm{~V})$ & $I(\mathrm{~A})$ & $P($ bars $)$ \\
\hline \multirow{3}{*}{ PA } & 0,75 & 520 & 890 & 3,8 \\
\cline { 2 - 5 } & \multicolumn{1}{|c|}{1} & 520 & 1080 & 4,2 \\
\hline \multirow{3}{*}{ PMMA } & 0,75 & 520 & 890 & 3,5 \\
\cline { 2 - 5 } & 1 & 465 & 1130 & 3 \\
\hline \multirow{3}{*}{ PU } & 0,75 & 520 & 1350 & 3,6 \\
\cline { 2 - 5 } & 1 & 450 & 1240 & 2,3 \\
\hline \multirow{3}{*}{ PAFV } & 0,65 & 520 & 980 & 4 \\
\cline { 2 - 5 } & 0,8 & 510 & 1060 & 3,3 \\
\cline { 2 - 5 } & 1 & 460 & 1250 & 3 \\
\hline \multirow{3}{*}{ PA 66 } & 0,55 & 770 & 500 & 3,1 \\
\cline { 2 - 5 } & 1 & 1260 & 430 & 2,8 \\
\hline
\end{tabular}

de la figure 12 qui représentent l'évolution du champ électrique en fonction de la largeur de fente. Le PAFV1 (sans colorant) présente, au vu de cette étude, des caractéristiques plus intéressantes (intensité du courant plus faible, champ électrique plus élevé).

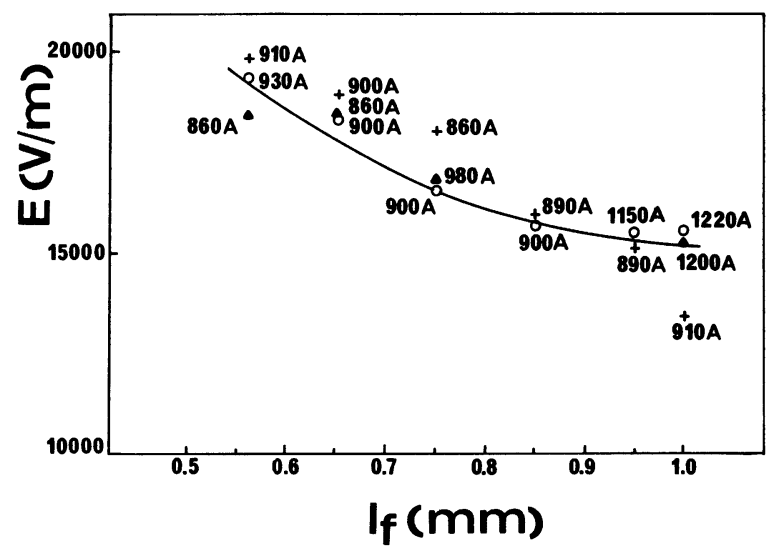

Fig: 11. - Variation du champ électrique en fonction de la largeur de la chambre pour différents polyacétals.

[Electric field variation according to the chamber width for different polyacetals.]

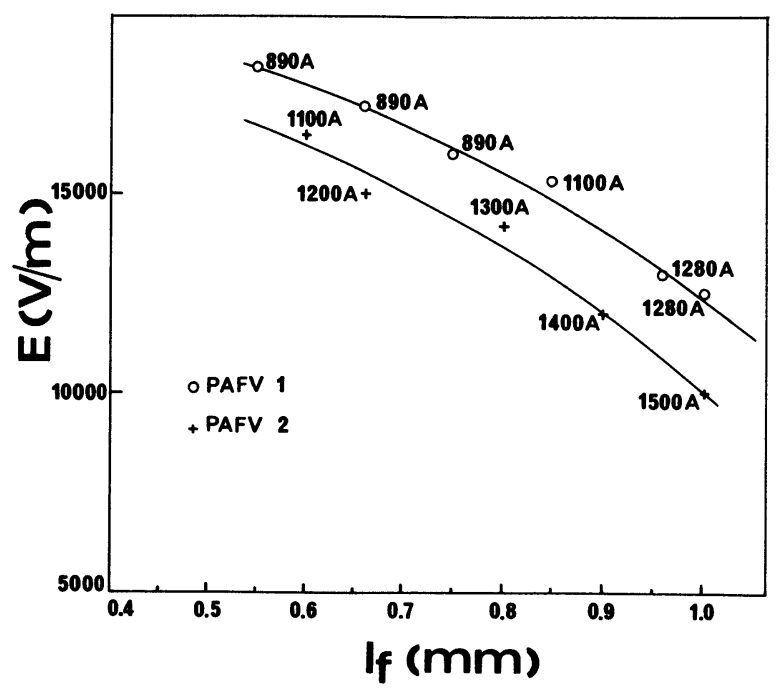

Fig. 12. - Variation du champ électrique en fonction de la largeur de la fente pour deux sortes de PAFV.

[Electric field variation according to the slit width for two sorts of PAFV.]

\section{Analyse des résultats.}

Les performances de l'appareillage de coupure dépendent d'une bonne limitation du courant d'arc et donc d'une valeur élevée du champ électrique.

L'influence de la largeur de fente a été établie pour tous les échantillons. Lorsqu'elle diminue, l'intensité du courant diminue et le champ électrique augmente. Ces phénomènes seront plus ou moins accentués selon les matériaux.

Parmi les matériaux testés, on peut considérer d'abord le PTFE et la céramique pour lesquels l'intensité du courant d'arc reste élevée même pour une faible largeur de la chambre de décharge. La similitude de comportement, entre ces deux matériaux de nature très différente, semble montrer que la conductivité thermique de la paroi $(0,25 \mathrm{~W} / \mathrm{m} . \mathrm{K}$ pour le PTFE ; $1,4 \mathrm{~W} / \mathrm{m} . \mathrm{K}$ pour la céramique) ne constitue pas un critère de choix.

Les autres matériaux expérimentés réduisent de manière importante l'intensité du courant d'arc pour les faibles largeurs de fente. L'évolution du champ électrique en fonction de la largeur de fente dépend du matériau. On peut distinguer :

- AR, PU, PA 6-6 dont la courbe $E\left(l_{\mathrm{f}}\right)$ présente un palier lorsque la largeur de fente diminue;

- les matériaux qui entraînent une augmentation régulière du champ électrique au fur et à mesure de la diminution de la largeur de fente ;

- le PMMA qui laisse prévoir de fortes valeurs du champ électrique pour des fentes très étroites.

$\mathrm{Au} \mathrm{vu}$ des seuls résultats concernant le champ électrique, c'est le meilleur des matériaux expérimentés. 
Lors de l'utilisation de ces matériaux à fort courant d'autres critères sont à considérer comme l'ablation des parois ou l'encrassement de la chambre.

Les caractéristiques de la décharge dépendent fortement de la nature du plasma d'arc. Ceci est confirmé par l'étude de l'influence des additifs.

La différence de comportement entre PAFV1 et 2 peut avoir pour origine la différence d'absorption d'énergie par deux matériaux de couleur différente mais il peut également être lié à la présence d'un colorant noir généralement à base de noir de carbone. La présence de carbone dans une décharge entraîne en effet une augmentation de la conductivité électrique ainsi que celle du temps d'extinction.

La comparaison des performances des matériaux PAFV1 et PA 6-6 confirme l'effet favorable des fibres de verre. En effet, la silice (utilisée dans les fusibles) est un bon extincteur d'arc.

L'influence des additifs ou composants sur les caractéristiques de la décharge peut être fortement augmentée lors de l'utilisation de ces matériaux à fort courant.

Pour certains échantillons on observe un phénomène de «traînage » et la durée d'arc reste notable. Il s'agit d'un polyacétal noir et dans certains cas de PAFV1. L'analyse des courbes de variation du champ électrique en fonction du temps montre un changement de régime d'arc au bout de $4 \mathrm{~ms}$. On peut en effet considérer deux régimes d'arc différents :

a) le milieu occupant le volume de la chambre de décharge est conducteur dans son ensemble, la densité de courant est faible, la valeur du champ électrique dépend de ce milieu qui est constitué de vapeurs d'isolants profondément modifiées pendant la première phase de la décharge ; b) l'arc comporte deux zones présentant des caractéristiques de conduction très différentes. Le volume conducteur au voisinage de l'axe est très réduit, la valeur de la densité de courant reste élevée comme celle du champ électrique. Le milieu environnant est alors constitué de vapeurs peu conductrices. C'est le modèle proposé par Niemeyer [2].

L'ensemble des résultats obtenus montre l'importance des caractéristiques du plasma formé lors de la décharge et la compréhension des phénomènes observés passe par la recherche des mécanismes qui régissent ce type d'arc.

\section{Conclusion.}

Les démarreurs et contacteurs sont destinés à opérer à des puissances de plus en plus grandes. Par ailleurs, leur miniaturisation conduit à des densités d'énergie très importantes. Dans plusieurs types d'appareillages le plasma d'arc est contaminé par des vapeurs provenant du matériau diélectrique constituant le boîtier. Un choix judicieux du matériau et de la géométrie peut donc permettre une amélioration du fonctionnement des appareils de coupure mais la recherche et l'utilisation d'interactions fortes entre l'arc et les parois des chambres de décharge conduit à la mise en œuvre d'appareillage de coupure de conception nouvelle. Comme le montre cette étude, l'extinction de l'arc dépend alors essentiellement du choix des matériaux.

\section{Remerciements.}

Cette étude a été réalisée au sein de la Société Télémécanique Electrique, l'auteur remercie pour son soutien constant la Direction Recherche et Développement.

\section{Bibliographie}

[1] Belbel E., Lauraire M., 13th Int. Conf. on Electric Contacts, Lausanne (1986) 150-155.

[2] Niemeyer L., I.E.E.E. Trans. Power Appar. Syst. PAS-97 (1978) 950-958.

[3] Ibrahim E. Z., J. Phys. D : Appl. Phys. 13 (1980) 2045-2065.

[4] Kovitya P., Lowke J. J., J. Phys. D : Appl. Phys. 17 (1984) $1197-1212$.
[5] Tslaf A., Proc. I.E.E.E. 24 (1977) 411-412.

[6] Tslaf A., I.E.E.E. Trans. Electr. Insul. EI-14 (1979) 51-57.

[7] Laurent A., Doctorat de 3ème Cycle, No. 706 (1983), Université Blaise Pascal, Clermont-Ferrand. 